\title{
Conditioned suppression in rats responding for hypothalamic brain stimulation
}

\author{
H. KENT MERRILL \\ Arizona State University, Tempe, Arizona 85281
}

\begin{abstract}
Rats were trained to press one lever on a variable interval schedule of reinforcement in order to produce a second lever. Each response on the' second lever produced a single burst of hypothalamic electrical stimulation, and following a fixed number of bursts the second lever was retracted. After stabilization of responding, fear conditioning was superimposed on the leverpressing situation. All of the rats developed complete suppression of responding within a few trials. The schedule of reinforcement then was systematically manipulated to increase either the density or the intensity of reinforcement by increasing the frequency with which the second lever was made available, increasing the number of reinforced responses allowed on the second lever, or increasing the intensity of the brain stimulation. Following each such manipulation, there was a temporary attenuation of suppression followed by the reappearance of suppression.
\end{abstract}

Many of the supposed anomalies of intracranial stimulation (ICS) have been demonstrated to be somewhat less than anomalous when the different procedures utilized in most ICS studies are taken into account. The supposedly rapid extinction of responding for ICS has been shown to be dependent on situational parameters (Gibson, Reid, Sakai, \& Porter, 1965; Olds \& Christenson, 1970); the reported insatiability of ICS may have been somewhat exaggerated (Bromley, 1967); and the alleged inability of ICS to maintain responding on intermittent schedules of reinforcement does not hold up in the face of empirical evidence (Merrill, Bromley, \& Porter, 1969; Pliskoff, Wright, \& Hawkins, 1965). Nevertheless, it has been reported consistently that animals responding for ICS are not affected by aversive stimulation as readily as animals responding for food or water (Brady \& Conrad, 1960; Geller, 1970; Goldstein, 1966; Merrill \& Anderson, 1970; Merrill, Lott, \& Bergen, 1970).

Most investigators of this phenomenon have utilized the standard ICS procedure of training rats to make single responses for short, single bursts of ICS. Geller (1970) did utilize a variable-interval schedule as part of a discrimination procedure involving footshock, but the presentation of footshock itself occurred when his subjects were receiving ICS for every response. Brady and Conrad (1960) used a variable-interval schedule, but when reinforcement contingencies were in effect, a single leverpress resulted in immediate delivery of a single short burst of ICS. As Pliskoff et al. (1965)

This research was supported in part by funds from the National Institute of Mental Health, Grant MH-17341-01, and in part by funds allocated to the author during his tenure as a Research Associate at the Veterans Administration Hospital, Phoenix, Arizona. The assistance of Donald Shearer, Charles E. Wilson, and Donovan E. Fleming is appreciated. Reprints may be obtained from the author, Department of Psychology, Arizona State University, Tempe, Arizona 85281. have pointed out, such conditions are not necessarily comparable to the standard operant situation where a rat working for food presses a lever and then performs a chain of approach and consummatory responses prior to ingestion. Pliskoff et al. (1965) and Merrill et al. (1969) proposed that the response of an animal pressing a lever which results in the immediate delivery of ICS may be a consummatory response rather than an instrumental response. Although consummatory responses per se are not necessarily resistant to conditioned suppression, it is possible that the combination of a high incentive and a consummatory-like response might produce resistance to conditioned suppression or passive avoidance. Indeed, Vogel and Spear (1966) found that, while conditioned suppression of licking could be obtained readily in rats trained to lick a tube for a 4\% sugar water solution, suppression was attenuated in animals licking for a $32 \%$ solution. This is in contrast to the fact that conditioned suppression is readily obtained in rats which have to perform an instrumental leverpressing response in order to obtain a $30 \%$ sugar water solution (e.g., Merrill et al., 1970). Therefore, in an attempt to separate the consummatory and instrumental aspects of responding for ICS, the present study utilized the two-bar schedule described by Pliskoff et al. (1965).

\section{METHOD}

\section{Subjects}

The subjects were five naive male 90- to 120-day-old LongEvans rats. They were housed individually and allowed free access to food and water.

\section{Surgery and Histology}

Bipolar stainless steel electrodes (Plastic Products Co., Roanoke, Virginia) were stereotaxically implanted in all animals while they were under sodium pentobarbital anesthesia $(40 \mathrm{mg} / \mathrm{kg})$. The electrodes were placed in the posterior medial forebrain bundle, $3.5 \mathrm{~mm}$ posterior to bregma, $1.5 \mathrm{~mm}$ lateral 
to the midline, and $8.5 \mathrm{~mm}$ below the surface of the skull. The skull was level between bregma and lambda.

Histological verification of the electrode sites was obtained following the completion of testing. The brains were embedded in celloidin, sectioned on a microtome, and serial sections were stained with cresyl violet and compared with the plates of a stereotaxic atlas (König \& Klippel, 1963, Figures 36-37).

\section{Apparatus}

The subjects were trained and tested in two test chambers, 9.5 in. wide $\times 9.5$ in. long $\times 12$ in. high $(24 \times 24 \times 34 \mathrm{~cm})$, each containing a fixed lever, a retractable lever, a pellet tray, and a $24-\mathrm{V}$ light mounted 2 in. $(5.1 \mathrm{~cm})$ above each lever. The centers of the levers were 2 in. $(5.1 \mathrm{~cm})$ from each side wall, and the pellet tray was centered between the levers. The chambers were constructed of Plexiglas with a stainless steel grid floor. They were located adjacent to each other on a table in a sound-attenuated room adjacent to the room containing the control apparatus. The end walls of the chambers were painted black, so that the rats were not in visual contact. The room lights were turned off, and the light above the work lever served as a houselight during each session.

The ICS circuitry was the same as that described by Merrill et al. (1970) and delivered a $60-\mathrm{Hz}$ sine wave to the subject. The intensities required to maintain consistent responding ranged from 50 to 100 microamperes.

For fear conditioning, the conditioned stimulus (CS) was the onset of a $60-\mathrm{W}$ light mounted $12 \mathrm{in} .(34 \mathrm{~cm})$ above the two test chambers, which had clear Plexiglas tops. The unconditioned stimulus (UCS) was the scrambled output of a powerstat delivering $690 \mathrm{~V}$ through a 330,000-ohm resistor connected in series with the rat. The ICS and footshock circuitry were isolated from each other by double-pole double-throw relays to prevent interaction between the circuits.

\section{Self-Stimulation Training}

Following the procedure described by Pliskoff et al. (1965), the rats were trained to press the fixed lever (work lever) in order to produce the retractable lever (reinforcement lever). Each response on the reinforcement lever produced a .3-sec burst of ICS and, following 10 such bursts, the reinforcement lever retracted. The schedule of presentation of the reinforcement lever was increased gradually from a schedule where every response on the work lever produced the reinforcement lever to a schedule where the reinforcement lever was produced only for a work-lever response after varying intervals of time. The mean interval of time was $30 \mathrm{sec}$, and this schedule was designated as VI $30 \mathrm{Rf} 10$ (for variable interval 30, reinforcement 10).

\section{Conditioned Suppression}

The rats were trained to respond on the VI $30 \mathrm{Rf} 10$ schedule for ICS and, after stabilization of responding on this schedule, fear conditioning was superimposed on the leverpressing situation. Three pairings of a 60 -sec CS and a 2 -sec UCS were presented during each daily $1-\mathrm{h}$ session. The CS was presented alone on the first day. The time of presentation was randomly determined each day, with the limitation that the onset of the CS never occurred while either subject was responding on the reinforcement lever, except in the two CRF conditions as explained below.

A suppression ratio was calculated for responses on the work lever for each presentation of the CS during the intermittent schedules and for responses on the reinforcement lever for the schedules where reinforcement was delivered for every response. The ratio was $\mathrm{B} / \mathrm{A}+\mathrm{B}$, with $\mathrm{A}$ representing the mean number of leverpresses per minute during the two 60 -sec periods immediately prior to the onset of the CS and B representing the number of leverpresses during the $60-\mathrm{sec} C S$. $\mathrm{A}$ ratio of .00 indicates complete suppression and a ratio of .50 indicates no suppression.
Following 2 days with a combined mean suppression ratio of .20 or less, the parameters of the schedule were changed so as to increase either the reinforcement density or the intensity of ICS. The animals were stabilized under the new conditions for 1 day and then fear conditioning again was superimposed on the new baseline. For two of the subjects (1 and 2), the successive changes in the schedule were: (1) VI $30 \mathrm{Rf} 10$; (2) VI $30 \mathrm{Rf} 50$, where 50 reinforced responses were allowed during each presentation of the reinforcement lever, with no change in ICS intensity; (3) CRF-low, where the reinforcement lever was available continuously and every response on it was reinforced with the same intensity of ICS as used previously; (4) CRF-high, where the reinforcement lever was available continuously and every response was reinforced, but with the intensity of the ICS increased by 50\%; and (5) VI $30 \mathrm{Rf} \mathrm{10,} \mathrm{at}$ the original intensity of ICS. The last condition was introduced after 5 to 6 days on CRF-high, whether or not a suppression ratio below .20 had been obtained, to test whether attenuation effects were occurring as a result of adaptation over time. The animals were not given a day of adaptation to the new schedule between the CRF-low and CRF-high conditions.

For the other three subjects $(3,4$, and 5), an FR 1 Rf 10 schedule, wherein every response on the work lever produced the reinforcement lever, was substituted for the VI 30 Rf 50 condition in the above sequence.

\section{RESULTS}

\section{Histology}

All of the electrodes were located in the region of the medial forebrain bundle at a level bounded by the posterior dorsomedial and ventromedial nuclei anteriorally, and the premamillary and posterior hypothalamic nuclei posteriorly. The electrodes were located on, or slightly dorsal to, an imaginary line running from the fornix to the lower edge of the internal capsule (König \& Klippel, 1963, Figures 36-38).

\section{Conditioned Suppression}

The suppression ratios for each animal are presented in Figure 1. Each vertical line represents the end of a schedule presentation. On the first day of testing, when the CS was presented alone, all of the animals showed adaptation to the CS. During the VI $30 \mathrm{Rf} 10$ segment of the procedure, all five rats developed total suppression to the CS-UCS pairings. Increasing the reinforcement density (VI $30 \mathrm{Rf} 50$ or FR $1 \mathrm{Rf} 10$ ) had no effect for one animal in each condition. However, each of the other rats showed varying degrees of temporary attenuation of suppression, with a great deal of inter- and intra-animal variation. When the intensity of the ICS was increased during CRF-high, suppression was once more attenuated and, in this case, although there was some degree of reacquisition for most animals, none showed complete suppression consistently under these conditions. However, the attenuation generally took the form indicated in the CRF-high portion of Figure 2. Note in Figure 1 that Trials 31-33 for Rat 5 produced suppression ratios of $.20, .23$, and .22. The cumulative record in Figure 2 shows that this partial suppression was the result of Rat 5 continuing 


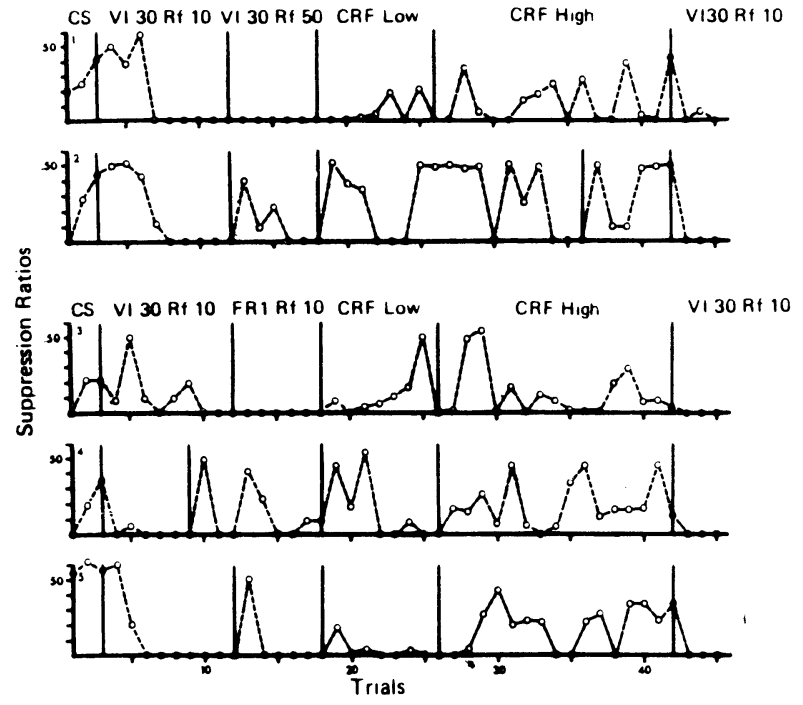

Figure 1. Suppression ratios during the various schedules of reinforcement. $\mathbf{C R F}=$ continuous reinforcement; $\mathbf{C S}=$ conditioned stimulus presented alone; $F R=$ fixed ratio; $R f=$ reinforcement component $; \mathrm{VI}=$ variable interval; Low $=$ low intensity ICS (the value used to maintain responding in all conditions except CRF-High); High = high intensity ICS (ICS increased $50 \%$ above the intensities used in all other conditions). Thus, VI 30 Rf $10=$ VI 30-sec schedule on the work lever with 10 reinforcements allowed on the reinforcement lever. The nomenclature and the details of the schedules are further explained in the text. Trials refer to conditioned suppression trials. Three such trials were presented each day, and one or more days of baseline training occurred between each schedule change (indicated by vertical lines). A suppression ratio of .00 indicates complete suppression (see text).

to respond throughout the first portion of the CS and then suppressing its responses completely as the time for UCS presentation approached. This pattern has been seen by us in other animals as well, although not in all. When returned to the VI 30 Rf 10 schedule, all animals showed complete suppression. Sample cumulative records from the various schedules are shown in Figure 2.

\section{DISCUSSION}

The readiness with which conditioned suppression appeared in the animals of this study stands in marked contrast to the lack of such suppression reported in previous studies (Brady \& Conrad, 1960; Goldstein, 1966; Merrill et al., 1970). Thus, it would appear that the attenuation of aversive stimulation by ICS may be classed with the other "anomalies" discussed in the introduction to this paper: It is anomalous primarily in that ICS is not food and, when procedures are effected which accommodate the differences in the nature of the reinforcers, similar functional properties emerge. In this case, separation of "instrumental" and "consummatory" aspects of responding for ICS during the initial VI $30 \mathrm{Rf} 10$ segment of the procedure produced rapid acquisition of conditioned suppression. When the "consummatory" component was tested for resistance to suppression (in the CRF segments), it proved resistant to suppression only at a high intensity of reinforcement, although the initial shift to CRF-low did produce a temporary attenuation of suppression. This is similar to the pattern found by Vogel and Spear (1966) in rats licking a tube for sugar water.

Two findings prevent us from concluding uncategorically that ICS and food are totally equivalent when procedural differences are eliminated. First, the worklever/reinforcement-lever procedure is not a sufficient condition for the production of suppression. Other studies in our laboratory indicate that in a situation where rats are pressing on a VI 30 Rf 10 schedule, but are fear conditioned in a chamber separate from the baseline leverpressing situation, attenuation of suppression does occur in some animals (Merrill, Wilson, \& Fleming, Note 3). Second, we have found that, when multiple schedules which produce matched response rates for food and ICS are in effect, nonresponsecontingent shock does suppress responding for ICS, but it does not suppress such responding as greatly as it does responding for food (McCaleb, Schmidt, \& Merrill, Note 1). Nevertheless, the results of the present study do indicate that responding for ICS is susceptible to conditioned suppression under some procedural conditions.

The temporary disappearance of suppression after each shift in reward conditions is compatible with an incentive interpretation of the suppression-attenuation phenomenon. The pattern of suppression shown in the CRF(b) condition of Figure 2 also would fit this

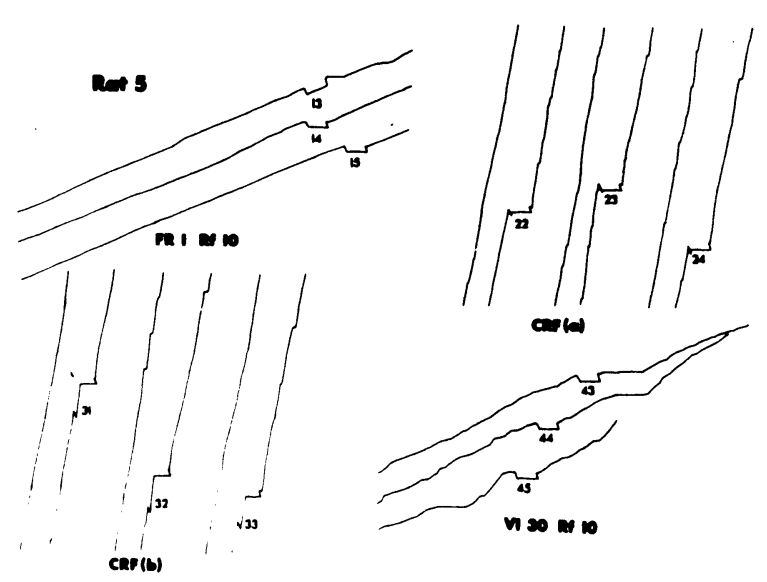

Figure 2. Typical cumulative records from the various reinforcement schedules for Rat 5 . The offset of the response pen indicates CS presentation, with UCS presentation immediately following the termination of the CS. The numbers below the offset of the pen are the trial numbers. For the FR 1 Rf 10 schedule and the $\mathrm{VI} 30 \mathrm{Rf} 10$ schedule, responses on the work lever are shown. For the two CRF schedules, responses on the reinforcement lever are shown. CRF(a) = low intensity ICS; CRF(b) = high intensity ICS. 
interpretation (the argument being that the "incentive" is high enough to attenuate fear during the early part of the CS, but then the "fear gradient" overcomes the "incentive gradient"). However, there are problems with such an interpretation. First, there was no independent measure of incentive in these procedures. Subsequent studies in our laboratory have attempted to provide an independent measure of incentive through the use of concurrent and multiple food and ICS schedules of reinforcement, but the results of those studies have not demonstrated conclusively that matching response rates also matches incentive values (McCaleb et al., Note 1; Merrill, Schmidt, \& McCaleb, Note 2). Nevertheless, it is difficult to conceive of a fivefold increase in the number of ICS bursts (as in the change from VI $30 \mathrm{Rf} 10$ to VI $30 \mathrm{Rf} 50$ ) as having no effect on incentive. Second, the attenuation after each change in schedule conditions could have been the result of stimulus change, or generalization decrement (Nevin, 1973). The first time the CS was presented on the new segment of the schedule, the rat might not have associated it with shock. Most of the one ${ }^{\iota}$ or two-trial attenuations might be accounted for by this explanation, but the general reduction of suppression in the CRFhigh segment and the immediate return to suppression in the last VI $30 \mathrm{Rf} 10$ segment would remain to be explained.

The fact that more suppression was obtained in the two-lever situation than in the one-lever situation might be due to attentional rather than motivational factors. If an animal is receiving an intensely reinforcing internal stimulus such as ICS, it may be less likely to attend to an external CS. However, we previously have demonstrated that rats which are not receiving ICS during the onset of the CS do still consistently show attenuation of conditioned suppression under certain conditions (Merrill et al., 1970, Note 2). Also, this explanation does not account for the temporary attenuation of suppression when reinforcement contingencies were increased in the two-lever paradigm. In that paradigm the experimenter manually controlled the onset of the CS and never presented it while the rats were responding on the reinforcement lever. In the FR 1 Rf 10 segment, there was very little time between presentations of the reinforcement lever, but CS onset always occurred during that brief interval. In the VI 30 segments there were much longer periods of time during which ICS was not being received. Thus, while differences in attentional states might account for some of the results, they do not account for all of them.

As is the case with previous studies in this area, this study does not explain the physiological mechanisms involved in the attenuation of aversive stimuli by ICS. Nevertheless, it does indicate that the instrumental aspect of responding for ICS is more vulnerable to conditioned suppression than the consummatory aspect. It also indicates that the effectiveness of conditioned suppression varies with changes in reinforcement magnitude and density. This is consistent with a motivational, or incentive, interpretation of the effects of ICS.

\section{REFERENCE NOTES}

1. M. McCaleb, E. Schmidt, \& H. K. Merill. Matched food and ICS responding differentially suppressed following footshock in rats. Paper presented at the Western Psychological Association meeting, April 1975, Sacramento, California.

2. H. K. Merrill, E. Schmidt, \& M. McCaleb. Attenuation of fear conditioning by reinforcing intracranial stimulation: Concurrent and multiple food-ICS schedules. Paper presented at the Rocky Mountain Psychological Association meeting, May 1975, Salt Lake City, Utah.

3. H. K. Merrill, C. E. Wilson, \& D. E. Fleming. Differential effects on- vs. off-baseline fear conditioning in rats responding for hypothalamic brain stimulation. Paper presented at the Western Psychological Association meeting, May 1972, Portland, Oregon.

\section{REFERENCES}

Brady, J. V., \& Conrad, D. C. Some effects of limbic system self-stimulation upon conditioned emotional behavior. Journal of Comparative and Physiological Psychology, 1960, 53, 128-137.

BROMLEY, B. L. Long-term availability of intracranial selfstimulation. Unpublished Master's thesis. University of Utah, 1967.

GELLER, I. Effect of punishment on lever pressing maintained by food reward or brain stimulation. Physiology and Behavior. 1970, 5, 203-206.

Gibson, W. E., Reid, L. D., Sakai, M., \& Porter, P. B. Intracranial reinforcement compared with sugar-water reinforcement. Science, 1965, 148, 1357-1359.

GoldSTEIN, R. Effects of noncontingent septal stimulation on the CER in the rat. Journal of Comparative and Physiological Psychology, 1966, 61, 132-135.

König, J. F. R., \& Klippel, R. A. The rat brain. Baltimore: Williams and Wilkins, 1963.

Merrill, H. K., \& Anderson, D. C. Attenuation of a passiveavoidance response via reinforcing intracranial stimulation in rats. Journal of Comparative and Physiological Psychology, 1970, 73, 274-277.

Merrill, H. K., Bromley, B. L., \& Porter, P. B. "Frustration" from withholding reinforcing intracranial stimulation. Physiology and Behavior, 1969, 4, 345-349.

Merrill, H. K., Lott, W. J., \& Bergen, B. J. Attenuation of a conditioned emotional response via reinforcing intracranial stimulation in rats. Journal of Comparative and Physiological Psychology, 1970, 71, 426-434.

Nevin, J. A. Stimulus control. In J. A. Nevin \& G. S. Reynolds (Eds.), The study of behavior. Glenview, Ill: Scott, Foresman, 1973. Pp. 115-152.

Olds, M. E., \& Christenson, T. Effects of drive and training on extinction after self-stimulation and food reward. American Journal of Physiology, 1970, 219, 208-213.

Pliskoff, S. S., Wright, J. E., \& Hawkins, T. D. Brain stimulation as a reinforcer: Intermittent schedules. Journal of the Experimental Analysis of Behavior, 1965, 8, 75-88.

VOGEL, J. R., \& SPEAR, N. E. Interaction of reward magnitude and conditioned fear on the consummatory response. Psychonomic Science, 1966, 5, 263-264.

(Received in Austin October 23, 1975.) 